\title{
Renal Complications Related to Checkpoint Inhibitors: Diagnostic and Therapeutic Strategies
}

\author{
Julie Belliere ${ }^{1,2,3, *}$, Julien Mazieres ${ }^{3,4,5}$, Nicolas Meyer ${ }^{3,4,6}$, Leila Chebane ${ }^{7}$ and Fabien Despas ${ }^{7,8}$ \\ 1 Department of Nephrology and Organ Transplantation, University Hospital of Toulouse, \\ 31400 Toulouse, France \\ 2 INSERM U1048, Institute of Metabolic and Cardiovascular Diseases, 31400 Toulouse, France \\ 3 Department of Biological Sciences, Paul Sabatier University, 31400 Toulouse, France; \\ mazieres.j@chu-toulouse.fr (J.M.); meyer.n@chu-toulouse.fr (N.M.) \\ 4 Institut Universitaire du Cancer Toulouse Oncopole, 31400 Toulouse, France \\ 5 Department of Pneumology, University Hospital of Toulouse, 31400 Toulouse, France \\ 6 Department of Dermatology, University Hospital of Toulouse, 31400 Toulouse, France \\ 7 Service Pharmacologie Médicale et Clinique, Centre Midi-Pyrénées de PharmacoVigilance, \\ de Pharmacoépidémiologie et d'Informations sur le Médicament, 31400 Toulouse, France; \\ Leila.chebane@univ-tlse3.fr (L.C.); fabien.despas@univ-tlse3.fr (F.D.) \\ 8 Service de Pharmacologie Médicale et Clinique, Faculté de Médecine, Université Paul Sabatier, \\ Equipe PEPSS Centre d'Investigation Clinique 1436, INSERM 1297, 31400 Toulouse, France \\ * Correspondence: belliere.j@chu-toulouse.fr
}

check for updates

Citation: Belliere, J.; Mazieres, J.; Meyer, N.; Chebane, L.; Despas, F. Renal Complications Related to Checkpoint Inhibitors: Diagnostic and Therapeutic Strategies.

Diagnostics 2021, 11, 1187. https:// doi.org/10.3390/diagnostics11071187

Academic Editors: Dil Sahali, Hassan Izzedine, Vincent Audard, Carole Henique, Mario Ollero and Henning Reis

Received: 28 February 2021

Accepted: 28 June 2021

Published: 30 June 2021

Publisher's Note: MDPI stays neutral with regard to jurisdictional claims in published maps and institutional affiliations.

Copyright: (C) 2021 by the authors. Licensee MDPI, Basel, Switzerland. This article is an open access article distributed under the terms and conditions of the Creative Commons Attribution (CC BY) license (https:// creativecommons.org/licenses/by/ $4.0 /)$.

\begin{abstract}
Immune checkpoint inhibitors (ICI) targeting CTLA-4 and the PD-1/PD-L1 axis have unprecedentedly improved global prognosis in several types of cancers. However, they are associated with the occurrence of immune-related adverse events. Despite their low incidence, renal complications can interfere with the oncologic strategy. The breaking of peripheral tolerance and the emergence of auto- or drug-reactive T-cells are the main pathophysiological hypotheses to explain renal complications after ICI exposure. ICIs can induce a large spectrum of renal symptoms with variable severity (from isolated electrolyte disorders to dialysis-dependent acute kidney injury (AKI)) and presentation (acute tubule-interstitial nephritis in $>90 \%$ of cases and a minority of glomerular diseases). In this review, the current trends in diagnosis and treatment strategies are summarized. The diagnosis of ICI-related renal complications requires special steps to avoid confounding factors, identify known risk factors (lower baseline estimated glomerular filtration rate, proton pump inhibitor use, and combination ICI therapy), and prove ICI causality, even after long-term exposure (weeks to months). A kidney biopsy should be performed as soon as possible. The treatment strategies rely on ICI discontinuation as well as co-medications, corticosteroids for 2 months, and tailored immunosuppressive drugs when renal response is not achieved.
\end{abstract}

Keywords: renal complications; acute kidney injury; immune check point inhibitors; immune-related adverse events

\section{Introduction}

Immune checkpoint inhibitors (ICIs) have been approved in the field of oncology, providing an original antitumor approach compared to chemotherapies. Their utilization relies on the drug's capacity to repair dysfunctional $\mathrm{T}$ cells resulting in the regression of various cancers. The "price to pay" is the risk of autoimmunity, leading to immune-related adverse events (irAEs) and, in some cases, end organ damage. The contributions of ICIs to kidney toxicity have been neglected and underestimated for several years, but it has now been acknowledged that they lead to acute kidney injury (AKI). This impacts renal function and, subsequently, oncologic treatment choices must be weighed. This review focuses on diagnostic and therapeutic strategies for ICI-related renal complications. 


\subsection{ICIs}

Both cytotoxic T-lymphocyte-associated protein 4 (CTLA-4) and programmed death 1 (PD-1) play a role as physiologic brakes on unrestrained cytotoxic T-effector function. CTLA-4 (CD 152) is a member of the B7/CD28 family. It mediates immunosuppression by indirectly diminishing signaling through the co-stimulatory receptor CD28. The CTLA4 blockade also restores $\mathrm{T}$ cell three-signal activation. Ipilimumab is the first and only FDAapproved CTLA-4 inhibitor. PD-1 is an inhibitory transmembrane protein expressed in T cells, B cells, natural killer cells, and myeloid-derived suppressor cells. Programmed deathligand 1 (PD-L1) is expressed on the surface of multiple tissue types, including many tumor cells and hematopoietic cells. PD-L2 is more restricted to hematopoietic cells. A blockade of the PD-1/PDL-1 pathway can enhance antitumor T cell reactivity and promote immune control over cancerous cells. Since the FDA approval of ipilimumab (human IgG1 k antiCTLA-4 monoclonal antibody) in 2011, eight more ICIs have been approved for cancer therapy. PD-1 inhibitors (pembrolizumab, nivolumab, cemiplimab) and PD-L1 inhibitors (atezolizumab, avelumab, and durvalumab) are on the current list of approved agents [1] Recent anti-CTLA4 antibodies such as tremelimumab and quavonlimab (MK-1308) are now used in combination with anti-PDL1. For example, a combination of the anti-CTLA4 tremelimumab and the anti-PDL1 durvalumab is promising in advanced non-small cell lung cancer [2], head and neck squamous cell carcinoma [3], and other solid tumors such as advanced hepatocellular carcinoma [4]. The use of quavonlimab in combination with pembrolizumab in first-line treatment has also been reported in advanced non-small-cell lung cancer [5] and advanced small-cell lung cancer [6]. Recent studies have identified several new immune checkpoint targets, such as lymphocyte activation gene-3 (LAG-3), T cell immunoglobulin and mucin-domain containing-3 (TIM-3), T cell immunoglobulin and ITIM domain (TIGIT), and V-domain Ig suppressor of T cell activation (VISTA) [7]. The studies have generated promising results in clinical trials. As reported in Table 1, the number of ICIs is increasing.

Table 1. ICI list with indications.

\begin{tabular}{|c|c|c|c|}
\hline ICI Class & Molecule & Date of Approval & Type of Indications \\
\hline anti-CTLA4 & $\begin{array}{l}\text { Ipilimumab } \\
\text { Tremelimumab } \\
\text { Quavonlimab }\end{array}$ & $\begin{array}{l}2011 \\
2015 \\
\text { current folder }\end{array}$ & $\begin{array}{l}\text { melanoma, renal cell carcinoma, CRC } \\
\text { mesothelioma, in combination with durvalumab in advanced } \\
\text { non-small cell lung cancer, head and neck squamous cell } \\
\text { carcinoma, advanced hepatocellular carcinoma }\end{array}$ \\
\hline anti-PD1 & $\begin{array}{l}\text { Pembrolizumab } \\
\text { Nivolumab } \\
\text { cemiplimab }\end{array}$ & $\begin{array}{l}2014 \\
2014 \\
2018\end{array}$ & $\begin{array}{l}\text { melanoma, hepatocellular carcinoma, cervical cancer, advanced } \\
\text { NSCLC, gastric cancers, Hodgkin lymphoma, primary } \\
\text { mediastinal large B-cell lymphoma, urothelial cancer, cutaneous } \\
\text { squamous cell carcinoma } \\
\text { melanoma, head and neck, hepatocellular carcinoma, renal cell } \\
\text { carcinoma, CRC, small lung cancer, advanced NSCLC } \\
\text { cutaneous squamous cell carcinoma }\end{array}$ \\
\hline anti-PDL1 & $\begin{array}{l}\text { Atezolizumab } \\
\text { Avelumab } \\
\text { Durvalumab }\end{array}$ & $\begin{array}{l}2016 \\
2017 \\
2017\end{array}$ & $\begin{array}{l}\text { advanced small cell lung cancer, advanced NSCLC, triple } \\
\text { negative breast cancer, urothelial cancer } \\
\text { Merkel cell carcinoma, urothelial cancer } \\
\text { urothelial cancer, locally advanced NSCLC, advanced SCLC }\end{array}$ \\
\hline anti-LAG3 & $\begin{array}{l}\text { Eftilagimod alpha } \\
\text { Relatlimab }\end{array}$ & $\begin{array}{l}\text { FDA approval March } 2020 \\
\text { current folder }\end{array}$ & $\begin{array}{l}\text { metastatic RCC, metastatic breast cancer, melanoma, advanced } \\
\text { NSCLC and head and neck squamous cell carcinoma } \\
\text { clinical trials recruiting }\end{array}$ \\
\hline anti-TIM3 & $\begin{array}{l}\text { TSR-022 } \\
\text { MBG453 } \\
\text { Sym023 } \\
\text { INCAGN2390 } \\
\text { LY3321367 } \\
\text { BMS-9862 } \\
\text { SHR- } 170258 \\
\text { RO7121661 }\end{array}$ & current folder & clinical trials recruiting \\
\hline
\end{tabular}


Table 1. Cont.

\begin{tabular}{|c|c|c|c|}
\hline ICI Class & Molecule & Date of Approval & Type of Indications \\
\hline anti-TIGIT & $\begin{array}{c}\text { Tiragolumab } \\
\text { MK-7684 } \\
\text { Etigilimab } \\
\text { BMS-986207 } \\
\text { AB-154 } \\
\text { ASP-8374 }\end{array}$ & $\begin{array}{l}\text { FDA approval January } 2021 \\
\text { current folder }\end{array}$ & $\begin{array}{l}\text { PD-L1-high non-small cell lung cancer } \\
\text { clinical trials recruiting }\end{array}$ \\
\hline anti-VISTA & $\begin{array}{l}\text { JNJ-61610588 } \\
\text { CA-170 }\end{array}$ & current folder & clinical trials recruiting \\
\hline anti-B7-H3 & Enoblituzumab & FDA approval December 2020 & Patients with Pretreated Metastatic HER2-Positive Breast Cancer \\
\hline
\end{tabular}

CRC: colorectal cancer; NSCLC: Non-small-cell lung carcinoma; SCLC: Small-cell lung carcinoma.

While therapy with this class of agents has resulted in improved clinical outcomes for patients with multiple tumor types, a broad spectrum of irAEs may affect any organ system, with variable clinical presentations.

\subsection{Incidence of Renal irAEs}

Although severe irAEs remain rare ( $10 \%$ of the cases under monotherapy), they can become life-threatening if not anticipated and managed appropriately [8]. The highest frequency has been observed with CTLA4 antibodies and combinations of ICIs. Global grade III and IV toxicities occur in $20 \%$ of patients. Renal toxicities are not the most frequent [9]: the incidence of AKI is $2 \%$ for ipilimumab, $1.9 \%$ for nivolumab, $1.4 \%$ for pembrolizumab, and $4.9 \%$ for the ipilimumab and nivolumab combination [10], but it is hypothesized that it will rise to between 9.9 and $29 \%$ in the near future [11]. The proportion of renal irAEs has not yet been detailed, which is why we carried out a search on the VigiBase Pharmacovigilance database. In February 2021, VigiBase ${ }^{\circledR}$ contained $>24$ million individual case safety reports (ICSRs) from 127 countries. Each ICSR consists of a description of the drugs that are suspected to cause adverse drug reactions and contains information on patient age, gender, medical history, country, drugs taken, and drug initiation and stop dates. As reported in Table 2, the proportion of renal ICSRs (classified by the System Organ Class "Renal and Urinary Disorders") ranged from 2.6 to $7.9 \%$.

Table 2. Incidence of renal disorders in individual case safety reports (ICSRs) of suspected adverse drug reactions, according to a recent search in the VigiBase Pharmacovigilance database.

\begin{tabular}{|c|c|c|c|}
\hline ICI Class & Drug Name & Number of ICSR, $n=$ & $\begin{array}{c}\text { Number of ICSR with Renal or } \\
\text { Urinary Adverse Effects } \\
n=(\%)\end{array}$ \\
\hline \multirow{3}{*}{ anti-CTLA4 } & $\begin{array}{c}\text { Ipilimumab } \\
\text { (alone or in combination) }\end{array}$ & 22,641 & $1021(4.5 \%)$ \\
\hline & $\begin{array}{c}\text { Ipilimumab } \\
\text { (combined with nivolumab) }\end{array}$ & 11,536 & $686(5.9 \%)$ \\
\hline & tremelimumab & 408 & $22(5.4 \%)$ \\
\hline \multirow{3}{*}{ anti-PD1 } & pembrolizumab & 29,633 & 1397 (4.7\%) \\
\hline & nivolumab & 51,705 & $2350(4.5 \%)$ \\
\hline & cemiplimab & 655 & $50(7.6 \%)$ \\
\hline \multirow{3}{*}{ anti-PDL1 } & atezolizumab & 8193 & $431(5.3 \%)$ \\
\hline & avelumab & 1300 & $82(6.3 \%)$ \\
\hline & durvalumab & 4372 & $116(2.7 \%)$ \\
\hline Anti-LAG3 * & relatlimab & 65 & $5(7.7 \%)$ \\
\hline anti-TIGIT & Tiragolumab & 8 & $0(0 \%)$ \\
\hline anti-B7-H3 & Enoblituzumab & 2 & $0(0 \%)$ \\
\hline
\end{tabular}


The Uppsala Monitoring Centre (UMC) receives individual case safety reports (ICSRs) of suspected ADRs sent by national pharmacovigilance centers, which are stored in the World Health Organization's (WHO) global safety database (VigiBase ${ }^{\circledR}$ ). In February 2021, VigiBase ${ }^{\circledR}$ contained $>24$ million ICSRs from 127 countries. Each ICSR consists of a description of the drugs that are suspected of causing ADRs and contains information on patient age, gender, medical history, country, drugs taken, and drug initiation and stop dates. Drugs are coded using the WHO drug dictionary, covering over 150,000 medicines and vaccines. The distribution of ICSRs is based on pharmacovigilance notifications sent by practitioners or patients. Therefore, the frequencies in the table are different from the overall incidence of adverse drugs reactions evaluated during clinical trials. The percentages in the table are used to assess the adverse drug reaction profile of each drug.

The distribution of ICSRs is based on pharmacovigilance notifications sent by practitioners or patients. Therefore, the frequencies in the table are different from the overall incidence of adverse drugs reactions evaluated during clinical trials. The percentages in the table are used to assess the adverse drug reaction profile of each drug. ${ }^{*}$ Eftilagim, no ICSR reported in VigiBase as of 11 June 2021.

\subsection{A Paradigm Shift from Renal "Toxicity"}

Contrary to conventional chemotherapies, ICIs can lead to renal injury through various mechanisms. When describing renal complications related to ICI exposure, one should be aware of a novel paradigm that involves deleterious indirect immune responses as opposed to direct toxicity, which is the case for numerous anticancer molecules [12].

There is no evidence of a dose-response relationship. Contrary to other drugs, ICIs are not excreted by glomerular filtration. They display the same pharmacokinetic properties as other therapeutic antibodies, which include little impact of kidney or liver function impairment. The dominant mechanism of ICI clearance remains proteolytic catabolism [13]. ICIs are distributed by means of diffusion and convection within tissues. The neonatal Fc receptor is responsible for the transport of ICIs back into the vascular system, which prevents the intracellular degradation of these drugs and, consequently, prolongs their halflife [14]. On the other hand, the generation of antibodies against ICIs increases clearance as well as receptor-mediated endocytosis. That is why the half-lives of ICIs are also quite long (6-27 days) and are affected by immune system determinants that increase interindividual variability [13].

Renal complications are mediated by immune responses with individual determinants. In fact, ICIs impact peripheral tolerance. Whereas CTLA4 signaling occurs in the tumordraining lymph nodes, PD1/PDL1 blockade occurs at the tissue level and in the tumor microenvironment. A recent special review [15] extensively describes several fundamental hypotheses evoked to explain ICI-related renal toxicities, including the implication of gut microbiome and immunosenescence pathways. As shown in Figure 1, (i) checkpoint inhibition could lead to the production of autoantibodies against self-antigens that share epitopes with tumors. This has been described for a lupus-like nephropathy that occurs after ipilimumab administration [16]. (ii) Checkpoint inhibition could drive the activity of self-reactive $\mathrm{T}$ cell clones. This was previously described in a case report of a patient presenting with fulminant myocarditis in which the selective clonal $\mathrm{T}$ cell populations infiltrating the myocardium were identical to those in tumors and skeletal muscle [17]. Regarding the kidneys, renal tubular cells express PDL1, which protects them from T cell-mediated autoimmunity. In fact, PDL1 is constitutively expressed on human cell line HK-2 cells and is dramatically up-regulated by inflammatory signaling by IFN-gamma for example [18]. Furthermore, PD-L1 is frequently expressed in various renal pathologies unrelated to ICI therapy and could be a prerequisite for susceptibility to developing AKI and deleterious immune-related AIN [19]. In addition, ICI-related nephritis is a rare event in renal cell carcinoma, but it may portend a higher likelihood of response. One possible explanation is antigenic overlap between normal tubular cells and tumor cells [20]. (iii) ICIs could lead to the reactivation of drug-specific $\mathrm{T}$ cells. The loss of tolerance for common 
drugs such as proton-pump inhibitors is suspected [21]. In brief, ICIs disrupt the peripheral immune tolerance between tubular cells, dormant auto-reactive $\mathrm{T}$ cells, and tolerogenic dendritic cells and promote the migration and activation of effector $\mathrm{T}$ cells in renal tissue. ICIs are also known to participate in pro-inflammatory cytokine release (mainly CXCL-10, TNF-alpha, and IL-6). This is why renal toxicity is very hard to predict and can occur after a long period.

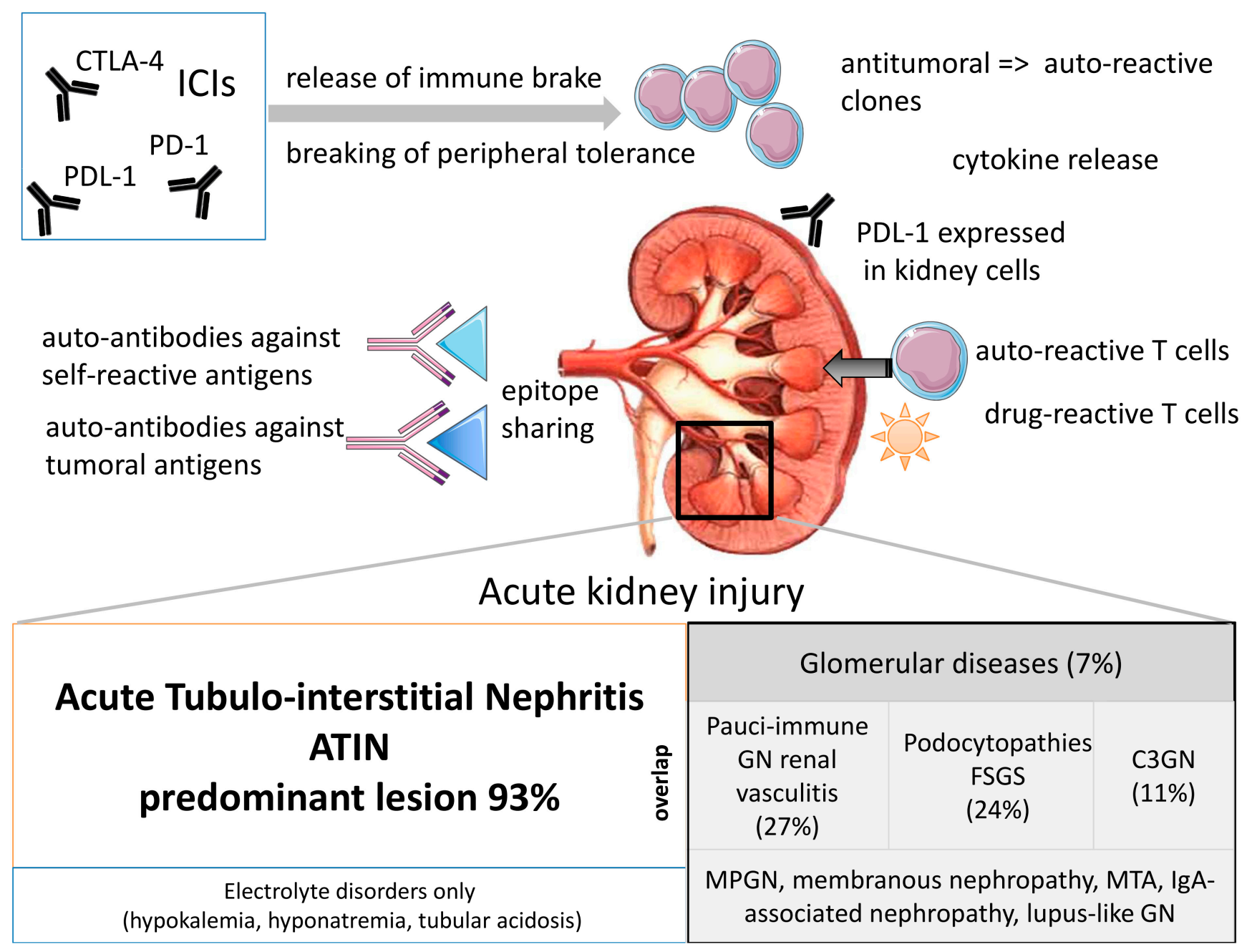

Figure 1. ICI-related renal complications: pathophysiology and landscape. While releasing the immune brake, ICIs lead to a disruption in peripheral tolerance. Through several mechanisms implicating both cellular and humoral immune responses, ICIs may lead to acute kidney injury. The predominant lesion is acute tubulo-interstitial nephritis (ATIN). Some glomerular diseases and electrolyte disorders have also been described.

The renal complications of ICIs encompass a wide landscape. In a multicentric study of 138 patients with ICI-associated AKI, defined as a two-fold increase in serum creatinine or dialysis requirement directly attributed to ICIs, acute tubulointerstitial nephritis (ATIN) was the dominant lesion in $93 \%$ of the 60 patients biopsied [22]. However, glomerular lesions have also been reported recently [23]: 45 cases of biopsy-confirmed ICI-associated glomerular disease were identified. Several lesion types were observed, the most frequent being pauci-immune glomerulonephritis (GN) and renal vasculitis (27\%) [24], podocytopathies (24\%) (minimal change disease [25], or FSGS [26]), and complement 3 GN (C3GN; $11 \%)$. Concomitant AIN was reported in $41 \%$ of patients. Other glomerular lesions have been observed [27], including IgA-associated glomerulonephritis (GN) [28], Goodpasture syndrome [29], membranoproliferative GN [30], lupus-like nephropathy [16], and thrombotic micro-angiopathy [31]. Furthermore, an overlap between ATIN and glomerular 
diseases can be noted (Figure 1). Interestingly, in some patients, renal lesions were only revealed by electrolyte disorders, including hyponatremia secondary to hypophysitis, hypokalemia [32], and distal renal tubular acidosis [33].

\section{Diagnostic Strategies}

Renal complications do not necessarily mean AKI. In some patients, ICI-related toxicity is revealed by only mild abnormalities such as isolated electrolyte disorders, or isolated urinalysis abnormalities (e.g., a single low-grade proteinuria at the early phase of podocyte injury). As patients with cancers have decreased muscle mass, and unlike conventional chemotherapeutics, ICIs do not cause classical drug nephrotoxicity, diagnosing ICI-related renal complications may be difficult. First of all, the conventional oncological approach with grade III to IV irAEs is inapplicable to renal function assessment. In the 2018 oncology guidelines [34], the interruption of ICIs and a consultation with the nephrologist were recommended when serum creatinine increased by a factor of $2-3$. It has been noted that even a rise that is $<1.5$ could be meaningful. However, according to the KDIGO staging and definition system for acute kidney injury, a $0.3 \mathrm{mg} / \mathrm{dL}$ increase within 48 hours is enough to assess AKI stage one [12] and to refer the patient to a nephrologist. The earlier the diagnosis of nephritis is made, the greater the chance of success with corticosteroids is.

We will now focus on the most frequent clinical situation, that is to say, the diagnosis of AKI in a patient with a history of ICI exposure, and illustrate the diagnostic strategy in a three-step process, summarized in Figure 2.

\section{- Diagnosis of suspected ICl-related AKI}

\section{1/ Assessment of clinical parameters}

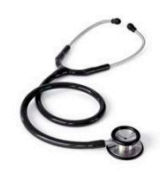

- platinum? anti-VEGF? iodine contrast? biphosphonates?

- renal lesions: urine culture, proteinuria, hematuria, AKI level

- extrarenal irAEs carefully screened in skin, thyroid, heart

- cytokine profiling, ICl dosages

- kidney imaging

\section{2/ In-depth assessment for suspected lesions}

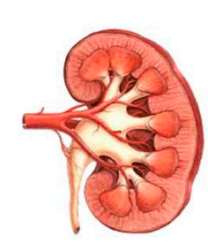

always if possible

diagnosis - infiltrate typing

prognosis

research (clones)

\section{BIOPSY}

\section{3/ Assessment for ICl causality}

extrinsic imputability: literature search // intrinsic imputability Intrinsic AKI with tubular, interstitial, or glomerular profile

Chronological score: 14 weeks after exposure; relapse if rechallenge Semiological score: associated irAEs, risk factors

Figure 2. Diagnostic strategy for AKI in a patient exposed to ICIs. When a patient presents with AKI, the clinician should assess whether it is ICI-related. This diagnosis can be difficult, which is why a careful strategy helps to elucidate the causal relationship between the patient's clinical and biological signs and the exposure to ICIs. PET scan: Positron emission tomography; usCD163: dosage of soluble CD163 level in urine. 


\subsection{First Step: Assessment for Clinical Renal Presentation}

The diagnostic strategy includes precise screening for the medical history (nephrological and oncological aspects), current and previous medications, as well as cardiovascular risk factors and habits. The patient has to be precisely questioned on recent events such as contrast CT scan, nephrotoxic drug exposure (angiotensin-converting enzyme inhibitor, angiotensin receptor blocker, non-steroidal anti-inflammatory drugs, proton pump inhibitors, bisphosphonates), dehydration, and screening for a systemic disorder (Sjögren's syndrome, Raynaud's syndrome, arthralgia, fever, digestive disorders, chest pain, urinary disorders).

Clinical examination focuses on signs of dehydration and possible extrarenal irAEs, especially cutaneous lesions. An extrarenal irAE, most often a rash, developed before or concomitant with AKI in $43 \%$ of the cases in a recent series [22].

Biological exams include a urinalysis for leukocyturia, hematuria, urine culture, as well as sodium/potassium ratio, magnesium, and sodium excretion fraction calculation, proteinuria, micro-albuminuria, and urine creatinine measurement. Given that some cases of ICI-related kidney toxicity may be restricted to isolated electrolyte disorders, clinicians should be aware of small variations in routine lab tests that suggest tubular dysfunction. A cystatin $C$ measurement could be very useful to confirm a decrease in the estimated glomerular filtration rate (eGFR) in patients receiving treatments that cause inhibition of renal transporters leading to a reversible and dose-dependent increase in creatinine [35]. In a series by Cortazar et al. on 138 patients, the urine protein-to-creatinine ratio was $>0.3 \mathrm{~g} / \mathrm{g}$ in $71 \%$ of the patients, urine dipstick was positive for leukocyte esterase, and pyuria was noted on the urine sediment in approximately half of the patients. None of these characteristics differed significantly according to AKI severity [22].

There should then be a systematic screening for the following other irAEs: thyroid disorders (TSH dosage), electrocardiogram, troponin, and BNP levels for cardiac injury, liver enzyme test, RBCs for associated hematological abnormalities (thrombocytopenia, TMA, hypereosinophilia), CPK (rhabdomyolysis), electrophoresis and immunofixation, and cytokine profiling (if available in the context of COVID). An ICI dosage should be performed if possible [36].

Finally, kidney imaging should be performed (echography or CT scan without contrast).

At this point, the clinician should be able to determine whether the patient suffers from pre-renal, post-renal, or intrinsic AKI and which renal compartment is involved in the ICI toxicity (tubulointerstitial, glomerular, or vascular origin).

\subsection{Second Step: In-Depth Assessment for Suspected Lesions}

If an ICI-related renal complication is suspected, the benefit/risk ratio for kidney biopsy for histological analysis should be discussed, with a multidisciplinary approach if possible, involving both oncologists and nephrologists. This discussion takes into account the feasibility of the procedure (kidney size, accessibility, coagulation disorders, anticoagulation retrieval), the patient's general health status, their choice and feelings about the overall situation, and most importantly, the possible therapeutic changes if the kidney biopsy is performed.

Whereas international guidelines do not recommend discussing kidney biopsy as a first-line investigative tool $[34,37]$, nephrologists oppose this practice. In a recent case series, five out of ten patients with suspected ICI-related AKI were found to have acute tubular injury/necrosis on biopsy [38]. This underlines the importance of proving acute tubular necrosis without an inflammatory component to avoid exposure to steroids. Furthermore, the identification of an associated lesion (e.g., a glomerular lesion such as vasculitis) as well as typing of immune infiltrates can impact the treatment choice to preserve future kidney function. Finally, a kidney biopsy provides precious information on baseline renal parenchyma through which a prognosis can be made. A kidney biopsy should be as representative as possible (including fixed and frozen sections, with a sufficient number of glomeruli) to allow routine staining and the detection of antibodies. Immunophenotyping of the immune infiltration in the kidney is mandatory to exclude lymphoproliferative 
disorders. Screening for T cell clones in the kidney could also be useful in some cases and requires a paraformaldehyde fixation to optimize their detection. T cell clones can be suspected in case of a positive membrane surface marker analysis (CD4, CD8). The pathologist should be able to specify whether the infiltrate is monomorphic or polymorphic. However, the confirmation of $\mathrm{T}$ cell clones depends on the results of a T-cell receptor gene rearrangement study. Expensive approaches such as ImmunoSEQ technologies can be considered. Kappa/lambda restriction orients more toward B cell clones.

When a kidney biopsy is not possible, non-invasive markers have been studied in preliminary works. Currently, there is no formal recommendation concerning this. Soluble urinary CD163 (suCD163) appears to be promising to reflect intra-renal infiltration by macrophages [15]. In fact, in a series of 72 cases of biopsy-proven acute tubular necrosis (ATN), an older age and a higher density of CD163+ macrophages predicted non-recovery, whereas the AKI stage, tubular injury score, and the density of CD68+ macrophage cells did not. The density of CD163+ M2 macrophages was an independent predictor of low eGFR at 3 months in advanced-stage AKI [39]. In ICI-related ATIN, some authors report the presence of CD163+ macrophages in kidney immune cell infiltration [40,41]. In various diseases associated with AKI, such as vasculitis and lupus nephropathies [42,43], suCD163 has been identified as a relevant marker. Prospective studies are needed to assess whether it plays a similar role in ICI-related AKI, especially when an invasive procedure is not possible. Recently, an increase in 18F-flourodeoxyglucose uptake in the renal cortex in a patient with checkpoint inhibitor-associated acute interstitial nephritis was described in a case report. This suggests that $18 \mathrm{~F}-$-flourodeoxyglucose positron-emission tomography could be a valuable diagnostic test for immune-mediated nephritis, particularly in patients where a timely kidney biopsy is not feasible [44].

\subsection{Third Step: Assessment for ICI Causality in Renal Lesions}

For frequent irAEs (hepatitis, colitis, and pneumonitis), a dedicated inpatient immune toxicity service (ITox) was established for patients admitted with irAEs using internal guidelines based on those of the NCCN and ASCO. Algorithms for defining an irAE as "definite", "likely", "possible", or "unlikely" were developed [45]. Unfortunately, such an approach has not yet been deployed for renal irAEs and it is the practitioner's responsibility to make this determination. Assessing the causality between the adverse events and suspected drugs is the most challenging task in pharmacovigilance. It requires close consideration of both the irAE and the suspected ICI, as well as patient-related factors, suspected concurrent drugs, and other medical conditions of the patient. Though different methods were developed to assess causality, no single method has been proven to produce an accurate or authentic ascertainable evaluation of the causal relationship [46]. However, the following arguments could be helpful:

- Extrinsic imputability: A literature search should be performed to identify similar cases.

- Intrinsic imputability with the following two criteria: (i) Chronological score: ICIrelated renal complications have a long latency period. In a series by Cortazar et al., the median (interquartile range) time from immune checkpoint inhibitor initiation to AKI was 14 (6-37) weeks [22] (as opposed to 4 weeks for skin diseases and 6 weeks for colitis). Practitioners should bear in mind that renal complications are possible even after the reintroduction of an ICI [24]. If a rechallenge is performed and AKI occurs again, the score is higher. (ii) Semiological score: Firstly, the patient exhibits known risk factors that have been previously established. A lower baseline eGFR, proton pump inhibitor use, and combination immune checkpoint inhibitor therapy were each independently associated with an increased risk of immune checkpoint inhibitor-associated AKI in the largest series [22]. The following other risk factors should be assessed: pembrolizumab and liver disease [47], as well as age $>65$ years. Secondly, the patient experiences or has recently experienced extrarenal irAEs in $40-87 \%$ of the cases (hypereosinophilia [48]; immune thrombocytopenic purpura [49]). 
On the contrary, the following arguments establish that the diagnosis of ICI-related renal complication requires confirmation: Previous exposure to platinum, pemetrexed [50], iodinated contrast, or a bisphosphonate. When an anti-VEGF has been used before the ICI, the interpretation of proteinuria kinetics is of major importance. Another possible bias is concomitant adrenalitis and adrenal insufficiency [51], leading to pre-renal AKI.

For renal transplant patients, distinctive features are testing for anti-HLA antibodies and $\mathrm{BK}$ virus nephropathy. ICIs could lead to very early graft rejection, graft intolerance syndrome, as well as cytokine storm, requiring graft nephrectomy [52] A kidney biopsy is also essential for diagnosis. A recent systematic review of twenty-seven articles with a total of 44 kidney transplant patients treated with ICI, reported a rejection rate of $40.9 \%$ [53]. The median time from ICI to a diagnosis of acute rejection was 24 (interquartile range, 10-60) days, which is shorter than the median time reported from ICI to AKI in non-transplant patients. The types of acute allograft rejection reported were cellular rejection (33\%), mixed cellular and antibody-mediated rejection (17\%), and an unspecified type (50\%). The percentage of allograft failure was high (88\%), and the mortality rate was $44 \%$ [53]. These data are similar to those published in another study that compares the rejection rate in several categories of solid-organ recipients. The highest rejection rate was noted in kidney transplant patients (40.1\%), followed by liver (35\%) and heart (20\%) transplant patients [54]. Recently, a disproportionality analysis of the VigiBase identified drugs associated with rejection. Kidney transplant rejection was associated with nivolumab (IC025 = 1.32), pembrolizumab $(\mathrm{IC} 025=1.17)$, and ipilimumab (IC025 = 0.33), which occurred in the same time frame (21 (interquartile range: 13; 56) days) [55]. In brief, T-cell mediated rejection with low participation of humoral response is the most frequent ICI-related complication in kidney transplant recipients, which is consistent with the suggested pathophysiology of ICI-related breaking of immune tolerance.

\section{Treatment Strategies}

Once it has been established that the renal complication is ICI-related, the treatment strategy should be rapid and efficient because it has now been acknowledged that AKI leads to an increased risk of chronic kidney disease. Whereas a decrease in renal function normally implies morbidity, it means mortality in oncology patients because most subsequent treatments require the highest possible level of renal function. Resolving an irAE can take precedence over the response of the cancer to the ICI. Recent treatment strategies for ICI-related renal complications are summarized in the following paragraph as well as Figure 3.

\subsection{Stop Exposure to ICI}

Although there are no published data on this subject, it is important to know that in life-threatening situations, the use of an antidote should be considered. This could be the case in patients experiencing fulminant myocarditis and AKI, considering that some authors have reported the successful use of plasma exchange (to remove circulating ICIs) and abatacept (to induce a co-stimulation blockade) [56].

In classical situations, supportive care should be initiated (renal replacement therapy: $9 \%$ in all ICI-related AKI patients [22], 25\% of patients with glomerular diseases [23]). Discontinuation of the ICI as well as suspected associated medications (PPI) is mandatory as soon as an irAE is suspected, to ensure timely implementation of the above-mentioned diagnostic strategy. In most instances, continuing treatment with ICI is not urgent because it is established that the benefit on the tumor lasts even after treatment is discontinued. Assessment by a nephrologist and a kidney biopsy, if performed, are not time-consuming from an oncological point of view. Usually, all aspects can be covered within a week, allowing the ICI to be delayed if necessary. 


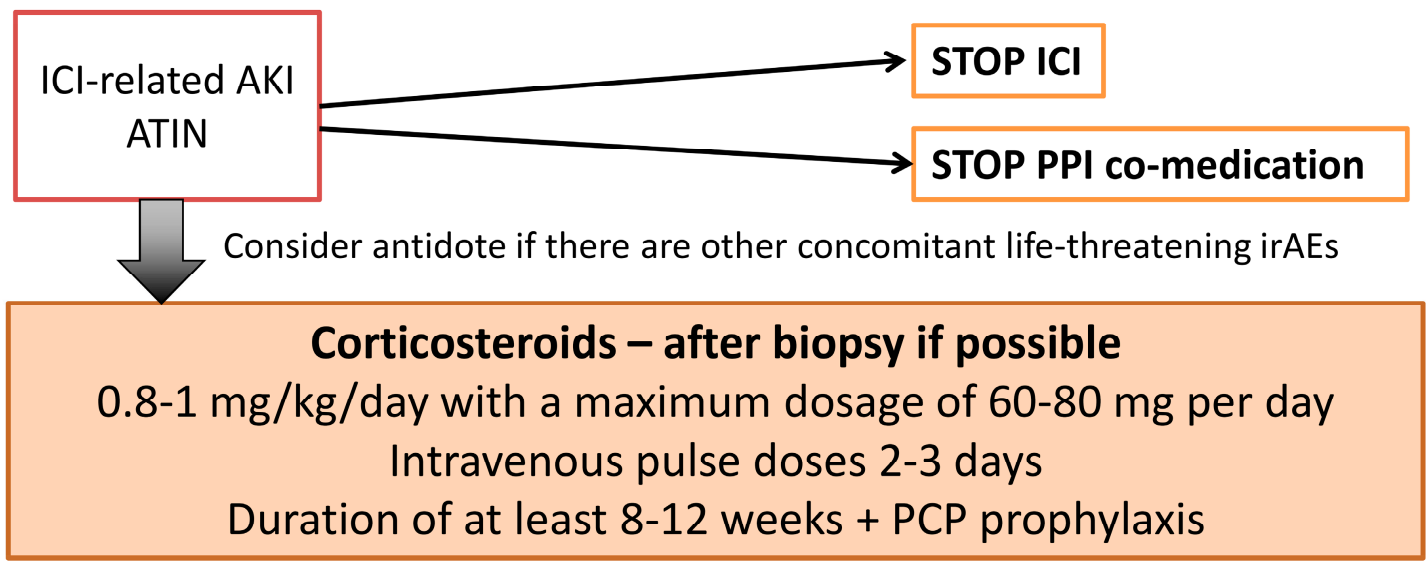

\section{Renal response?}

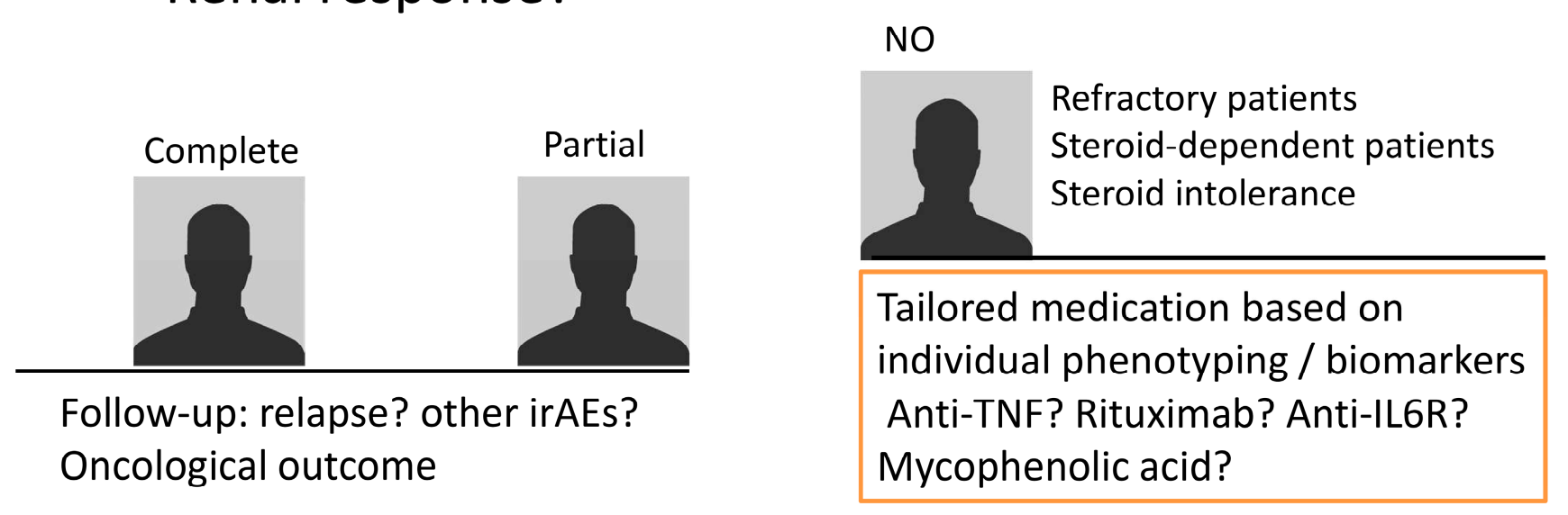

Figure 3. Therapeutic management for ICI-related renal toxicities When a patient is diagnosed with ICI-related ATIN, the therapeutic strategy is based on the discontinuation of ICI and co-medications. Corticosteroids are then the standard of care. Depending on the renal response, tailored medications based on individual phenotyping should be discussed. ATIN: acute tubulointerstitial nephritis; PPI: proton pump inhibitors; irAES immune related adverse events. PCP: pneumocystosis.

\subsection{Stop Immune Response Triggered by ICI}

In the prototypical ATIN situation, as well as in numerous irAEs, corticosteroids are the standard of care for ICI-related complications. There is no precise recommended protocol. However, experts agree to advocate $0.8-1 \mathrm{mg} / \mathrm{kg} /$ day with a maximum dosage of $60-80 \mathrm{mg}$ per day [57]. Pulse intravenous doses could be administered for 2-3 days, especially if oral absorption is not safe because of associated digestive irAEs. A kidney biopsy should not delay the initiation of corticosteroids. As might be expected, if corticosteroids have been administered for a long period before histological analysis ( $\sim 7$ days), the hypothesis of a "wash out" of infiltrating cells should be considered. The duration of corticosteroid therapy is not consensual, but a protocol of at least $8-12$ weeks is suggested, especially since ICIs can remain bound to the circulating lymphocytes for up to 57 days, with a mean plateau occupancy of $72 \%$ [58]. Furthermore, a higher dose of corticosteroids has been associated with a better prognosis [53].

As usual, corticosteroid initiation should take into account the risks of complications (diabetes, infections). Some authors mention the use of $\mathrm{PCP}$ prophylaxis with trimethoprimsulfamethoxazole. However, the risk of introducing a new medication frequently associated with ATIN should be further evaluated. A delayed introduction, when creatinine kinetics are favorable, might be an option to consider. 


\subsection{Tailor Immunosuppression to the Patient}

After ICI discontinuation and corticosteroid initiation, it is of the utmost importance to assess the quality of the renal response to first-line treatment. In a series by Cortazar et al., complete, partial, or no kidney recovery occurred in $40 \%, 45 \%$, and $15 \%$ of the patients, respectively. The failure to achieve kidney recovery after immune checkpoint inhibitor-associated AKI was independently associated with higher mortality. Concomitant extrarenal immune-related adverse events were associated with a worse renal prognosis, whereas concomitant tubulointerstitial nephritis-causing medications and treatment with corticosteroids were each associated with improved renal prognosis [22]. For patients with glomerular diseases, renal replacement therapy (RRT) was required in $25 \%$ of cases. Most patients had a full $(31 \%)$ or partial $(42 \%)$ recovery from an AKI, although $19 \%$ remained dialysis-dependent and approximately one-third died. A complete or partial remission of proteinuria was achieved in 45 and $38 \%$ of the patients, respectively [23].

\subsubsection{Refractory Patients}

In an era of evidence-based medicine and omics, we would like to tailor the treatment of ICI-related renal complications to the patient's individual situation. If a cytokine measurement revealed an associated cytokine increase, specific therapies should be considered (tocilizumab in the case of IL-6 increase). If the ICI dosages indicate high circulating levels, plasma exchanges can be initiated. A kidney biopsy should be performed again to provide additional indications to move to target therapies [59], especially since failure to achieve kidney recovery after an ICI-associated AKI is independently associated with a higher mortality.

3.3.2. Steroid-Dependent Patients or Patients with an Intolerance to Prolonged Steroid Schemes

For these patients, a sparing regimen has to be determined. However, very few data are available for renal irAEs. Data on mycophenolic acid are controversial: deleterious in patients with ATIN leading to pancytopenia and fatal septic complications [60], beneficial in patients with FSGS [61]. Rituximab should have a place in the treatment of ICI-induced vasculitis, as recently reported [62]. Anti-TNF alpha drugs are widely used in digestive irAEs, but there are no indications for renal complications. Future studies are needed to define a clear second-line strategy for patients with complicated ICI-related ATIN.

\subsection{Follow the Patient}

Due to their mechanisms of action, ICI-induced delayed immune responses explain delayed renal complications. The risk of relapse is present, but the incidence has not yet been quantified [63]. In some cases, we can hypothesize that the patient is still exposed to an immune trigger (co-medication). Attention should be paid to the occurrence of the other irAEs, and the patient should be carefully examined every month.

\subsection{Prevent Relapse If ICI Must Be Re-Started: The "Rechallenge"}

If ICIs are the only therapeutic option, the occurrence of a renal complication cannot prevent the rechallenge. As might be expected, these therapeutic choices are subjected to a consultation with nephrologists, oncologists, and the patient. The grade and history of the irAEs are of great importance (for example, in case of ICI-induced myocarditis a rechallenge is strictly forbidden). In a series by Cortazar et al. concerning ICI-related $\mathrm{AKI}$, the rechallenge occurred in $22 \%$ of the patients, $23 \%$ of whom developed recurrent associated AKI. Forty percent received corticosteroids (prednisone, 10-20 mg daily) in parallel. No data are available on class switching. A key point for the rechallenge is the identification of any drug associated with the first AKI episode. In fact, the prognosis is better if the co-medication has been interrupted. 


\subsection{Prevent the Disease in Future Patients}

Predictive factors for renal complications after ICIs are still lacking. It is unknown whether it might be related to the type of malignancy. Before ICI initiation, it is recommended to perform a precise renal examination with data on urinalysis and eGFR, avoid PPI use, and plan a precise follow-up for early detection of any renal complications. In case of solid organ transplant recipients (SOT), minimization of calcineurin inhibitors (CNIs) and the conversion of CNI to mTOR inhibitors (imTORs) along with judicious use of prophylactic steroids could enable the safe use of ICIs in patients with advanced cutaneous squamous cell carcinoma [64]. Clinicians should also be aware of the possibility of renal graft rejection, even in failed allografts [65].

\section{Conclusions}

The renal complications of ICIs still present numerous challenges for onconephrologists. The aim of having the best level of renal function presupposes early detection and recognition of renal complications, adequate biopsies, and rapid treatment. The impact of ICI-related AKI on the patient's global outcome remains to be defined.

Funding: This research received no external funding.

Acknowledgments: To Dil Sahali for his kind invitation to write this review, to Faguer and Ribes for the initiation of the project.

Conflicts of Interest: J.B.: none; J.M.: Research funding from Roche, AstraZeneca, and Pierre Fabre. Consulting/advisory role for AbbVie, Amgen, AstraZeneca, Bristol-Myers Squibb, Daiichi, Genentech/Roche, GlaxoSmithKline, Merck, MSD, Pfizer, Pierre Fabre, and Takeda.; N.M.: Investigator and/or consultant for BMS, MSD, Merck, Sanofi, Novartis, Roche, Pierre Fabre, Sun pharma, and Abbvie; L.C.: none; F.D.: none.

\section{References}

1. Brennan, D.C.; Aguado, J.M.; Potena, L.; Jardine, A.G.; Legendre, C.; Saemann, M.D.; Mueller, N.J.; Merville, P.; Emery, V.; Nashan, B. Effect of maintenance immunosuppressive drugs on virus pathobiology: Evidence and potential mechanisms. Rev. Med. Virol. 2013, 23, 97-125. [CrossRef]

2. Rizvi, N.A.; Cho, B.C.; Reinmuth, N.; Lee, K.H.; Luft, A.; Ahn, M.J.; Van Den Heuvel, M.M.; Cobo, M.; Vicente, D.; Smolin, A.; et al. Durvalumab with or without tremelimumab vs standard chemotherapy in first-line treatment of metastatic non-small cell lung cancer: The MYSTIC phase 3 randomized clinical trial. JAMA Oncol. 2020, 6, 661-674. [CrossRef] [PubMed]

3. Wang, B.-C.; Li, P.-C.; Fan, J.-Q.; Lin, G.-H.; Liu, Q. Durvalumab and tremelimumab combination therapy versus durvalumab or tremelimumab monotherapy for patients with solid tumors. Medicine 2020, 99, e21273. [CrossRef] [PubMed]

4. Kelley, R.; Kudo, M.; Harris, W.; Ikeda, M.; Okusaka, T.; Kang, Y.; Qin, S.; Tai, D.; Lim, H.; Yau, T.; et al. O-6 The novel regimen of tremelimumab in combination with durvalumab provides a favorable safety profile and clinical activity for patients with advanced hepatocellular carcinoma. Ann. Oncol. 2020, 31, 233-234. [CrossRef]

5. Perets, R.; Bar, J.; Rasco, D.W.; Ahn, M.J.; Yoh, K.; Kim, D.W.; Nagrial, A.; Satouchi, M.; Lee, D.H.; Spigel, D.R.; et al. Safety and efficacy of quavonlimab, a novel anti-CTLA-4 antibody (MK-1308), in combination with pembrolizumab in first-line advanced non-small-cell lung cancer. Ann. Oncol. 2021, 32, 395-403. [CrossRef] [PubMed]

6. Cho, B.C.; Yoh, K.; Bar, J.; Nagrial, A.; Spigel, D.R.; Gutierrez, M.; Kim, D.-W.; Kotasek, D.; Rasco, D.; Niu, J.; et al. Results from a phase I study of MK-1308 (ANTI-CTLA-4) plus pembrolizumab in previously treated advanced small cell lung cancer. Ann Oncol. 2019, 30, xi36-xi37. [CrossRef]

7. Qin, S.; Xu, L.; Yi, M.; Yu, S.; Wu, K.; Luo, S. Novel immune checkpoint targets: Moving beyond PD-1 and CTLA-4. Mol. Cancer 2019, 18, 1-14. [CrossRef] [PubMed]

8. Champiat, S.; Lambotte, O.; Barreau, E.; Belkhir, R.; Berdelou, A.; Carbonnel, F.; Cauquil, C.; Chanson, P.; Collins, M.; Durrbach, A.; et al. Management of immune checkpoint blockade dysimmune toxicities: A collaborative position paper. Ann. Oncol. 2016, 27, 559-574. [CrossRef]

9. Seethapathy, H.; Zhao, S.; Chute, D.F.; Zubiri, L.; Oppong, Y.; Strohbehn, I.; Cortazar, F.B.; Leaf, D.E.; Mooradian, M.J.; Villani, A.C.; et al. The incidence, causes, and risk factors of acute kidney injury in patients receiving immune checkpoint inhibitors. Clin. J. Am. Soc. Nephrol. 2019, 14, 1692-1700. [CrossRef]

10. Perazella, M.A.; Shirali, A.C. Immune checkpoint inhibitor nephrotoxicity: What do we know and what should we do? Kidney Int. 2020, 97, 62-74. [CrossRef] 
11. Wanchoo, R.; Karam, S.; Uppal, N.N.; Barta, V.S.; Deray, G.; Devoe, C.; Launay-Vacher, V.; Jhaveri, K.D. Cancer and Kidney International Network Workgroup on immune checkpoint inhibitors: Adverse renal effects of immune checkpoint inhibitors: A narrative review. Am. J. Nephrol. 2017, 45, 160-169. [CrossRef]

12. Rosner, M.H.; Jhaveri, K.D.; McMahon, B.A.; Perazella, M.A. Onconephrology: The intersections between the kidney and cancer. Cancer J. Clin. 2021, 71, 47-77. [CrossRef]

13. Centanni, M.; Moes, D.J.A.R.; Trocóniz, I.F.; Ciccolini, J.; van Hasselt, J.G.C. Clinical pharmacokinetics and pharmacodynamics of immune checkpoint inhibitors. Clin. Pharmacokinet. 2019, 58, 835-857. [CrossRef] [PubMed]

14. Keizer, R.J.; Huitema, A.D.R.; Schellens, J.H.M.; Beijnen, J.H. Clinical pharmacokinetics of therapeutic monoclonal antibodies. Clin. Pharmacokinet. 2010, 49, 493-507. [CrossRef] [PubMed]

15. Franzin, R.; Netti, G.S.; Spadaccino, F.; Porta, C.; Gesualdo, L.; Stallone, G.; Castellano, G.; Ranieri, E.; Ghezzi, P.; Saxena, A.; et al. The use of immune checkpoint inhibitors in oncology and the occurrence of AKI: Where do we stand? Front. Immunol. 2020, 11, 574271. [CrossRef] [PubMed]

16. Fadel, F.; El Karoui, K.; Knebelmann, B. Anti-CTLA4 antibody-induced lupus nephritis. N. Engl. J. Med. 2009, 361, 211-212. [CrossRef]

17. Johnson, D.B.; Balko, J.M.; Compton, M.L.; Chalkias, S.; Gorham, J.; Xu, Y.; Hicks, M.; Puzanov, I.; Alexander, M.R.; Bloomer, T.L.; et al. Fulminant myocarditis with combination immune checkpoint blockade. N. Engl. J. Med. 2016, 375, 1749-1755. [CrossRef] [PubMed]

18. Ding, H.; Wu, X.; Gao, W. PD-L1 is expressed by human renal tubular epithelial cells and suppresses T cell cytokine synthesis. Clin. Immunol. 2005, 115, 184-191. [CrossRef]

19. Hakroush, S.; Kopp, S.B.; Tampe, D.; Gersmann, A.K.; Korsten, P.; Zeisberg, M.; Tampe, B. Variable expression of programmed cell death protein 1-Ligand 1 in kidneys independent of immune checkpoint inhibition. Front. Immunol. 2021, 11, 2021. [CrossRef]

20. Patel, V.; Elias, R.; Formella, J.; Schwartzman, W.; Christie, A.; Cai, Q.; Malladi, V.; Kapur, P.; Vazquez, M.; McKay, R.; et al. Acute interstitial nephritis, a potential predictor of response to immune checkpoint inhibitors in renal cell carcinoma. J. Immunother. Cancer 2020, 8, e001198. [CrossRef]

21. Koda, R.; Watanabe, H.; Tsuchida, M.; Iino, N.; Suzuki, K.; Hasegawa, G.; Imai, N.; Narita, I. Immune checkpoint inhibitor (nivolumab)-associated kidney injury and the importance of recognizing concomitant medications known to cause acute tubulointerstitial nephritis: A case report. BMC Nephrol. 2018, 19, 48. [CrossRef] [PubMed]

22. Cortazar, F.B.; Kibbelaar, Z.A.; Glezerman, I.G.; Abudayyeh, A.; Mamlouk, O.; Motwani, S.S.; Murakami, N.; Herrmann, S.M.; Manohar, S.; Shirali, A.C.; et al. Clinical features and outcomes of immune checkpoint inhibitor-associated AKI: A multicenter study. J. Am. Soc. Nephrol. 2020, 31, 435-446. [CrossRef] [PubMed]

23. Kitchlu, A.; Jhaveri, K.D.; Wadhwani, S.; Deshpande, P.; Harel, Z.; Kishibe, T.; Henriksen, K.; Wanchoo, R. A systematic review of immune checkpoint inhibitor-associated glomerular disease. Kidney Int. Rep. 2021, 6, 66-77. [CrossRef] [PubMed]

24. Mulroy, M.; Ghafouri, S.; Sisk, A.; Ribas, A.; Goshtaseb, R.; Cherry, G.; Shen, J. Acute interstitial nephritis and PR3-ANCA following reintroduction of pembrolizumab: A case report. Immunotherapy 2021, 13, 283-288. [CrossRef] [PubMed]

25. Vaughan, E.; Connolly, E.; Hui, M.; Chadban, S. Minimal change disease in a patient receiving checkpoint inhibition: Another possible manifestation of kidney autoimmunity? Cancer Rep. 2020, 3, e1250. [CrossRef] [PubMed]

26. Glutsch, V.; Grän, F.; Weber, J.; Gesierich, A.; Goebeler, M.; Schilling, B. Response to combined ipilimumab and nivolumab after development of a nephrotic syndrome related to PD-1 monotherapy. J. Immunother. Cancer 2019, 7, 181. [CrossRef]

27. Mamlouk, O.; Selamet, U.; Machado, S.; Abdelrahim, M.; Glass, W.F.; Tchakarov, A.; Gaber, L.; Lahoti, A.; Workeneh, B.; Chen, S.; et al. Nephrotoxicity of immune checkpoint inhibitors beyond tubulointerstitial nephritis: Single-center experience. J. Immunother. Cancer 2019, 7, 2. [CrossRef]

28. Tanabe, K.; Kanzaki, H.; Wada, T.; Nakashima, Y.; Sugiyama, H.; Okada, H.; Wada, J. Nivolumab-induced IgA nephropathy in a patient with advanced gastric cancer: A case report. Medicine 2020, 99, e20464. [CrossRef]

29. Takahashi, N.; Tsuji, K.; Tamiya, H.; Shinohara, T.; Kuroda, N.; Takeuchi, E. Goodpasture's disease in a patient with advanced lung cancer treated with nivolumab: An autopsy case report. Lung Cancer 2018, 122, 22-24. [CrossRef]

30. Cruz-Whitley, J.; Giehl, N.; Jen, K.-Y.; Young, B. Membranoproliferative glomerulonephritis associated with nivolumab therapy. Case Rep. Nephrol. 2020, 2020, 2638283. [CrossRef]

31. Cortazar, F.B.; Marrone, K.A.; Troxell, M.L.; Ralto, K.M.; Hoenig, M.P.; Brahmer, J.R.; Le, D.T.; Lipson, E.J.; Glezerman, I.G.; Wolchok, J.; et al. Clinicopathological features of acute kidney injury associated with immune checkpoint inhibitors. Kidney Int. 2016, 90, 638-647. [CrossRef] [PubMed]

32. Balakrishna, P.; Villegas, A. Hypokalemic paralysis secondary to immune checkpoint inhibitor therapy. Case Rep. Oncol. Med. 2017, 2017, 5063405. [CrossRef] [PubMed]

33. El Bitar, S.; Weerasinghe, C.; El-Charabaty, E.; Odaimi, M. Renal tubular acidosis an adverse effect of PD-1 inhibitor immunotherapy. Case Rep. Oncol. Med. 2018, 2018, 1-3. [CrossRef] [PubMed]

34. Brahmer, J.R.; Lacchetti, C.; Schneider, B.J.; Atkins, M.B.; Brassil, K.J.; Caterino, J.M.; Chau, I.; Ernstoff, M.S.; Gardner, J.M.; Ginex, P.; et al. Management of immune-related adverse events in patients treated with immune checkpoint inhibitor therapy: American society of clinical oncology clinical practice guideline. J. Clin. Oncol. 2018, 36, 1714-1768. [CrossRef] 
35. Bruin, M.A.C.; Korse, C.M.; van Wijnen, B.; de Jong, V.M.T.; Linn, S.C.; van Triest, B.; Rosing, H.; Beijnen, J.H.; van den Broek, D.; Huitema, A.D.R. A real or apparent decrease in glomerular filtration rate in patients using olaparib? Eur. J. Clin. Pharmacol. 2021, 77, 179-188. [CrossRef] [PubMed]

36. Zalba, S.; Contreras-Sandoval, A.M.; Martisova, E.; Debets, R.; Smerdou, C.; Garrido, M.J. Quantification of pharmacokinetic profiles of pd-1/pd-11 antibodies by validated elisas. Pharmaceutics 2020, 12, 595. [CrossRef]

37. Thompson, J.A.; Schneider, B.J.; Brahmer, J.; Andrews, S.; Armand, P.; Bhatia, S.; Budde, L.E.; Costa, L.; Davies, M.; Dunnington, D.; et al. Management of immunotherapy-related toxicities, version 1.2019. J. Natl. Compr. Cancer Netw. 2019, 17, 255-289. [CrossRef]

38. Izzedine, H.; Mathian, A.; Champiat, S.; Picard, C.; Mateus, C.; Routier, E.; Varga, A.; Malka, D.; Leary, A.; Michels, J.; et al. Renal toxicities associated with pembrolizumab. Clin. Kidney J. 2019, 12, 81-88. [CrossRef] [PubMed]

39. Kim, M.G.; Lim, K.; Lee, Y.J.; Yang, J.; Oh, S.W.; Cho, W.Y.; Jo, S.K. M2 macrophages predict worse long-term outcomes in human acute tubular necrosis. Sci. Rep. 2020, 10, 1-7. [CrossRef]

40. Okawa, S.; Fujiwara, K.; Shimonishi, A.; Matsuura, H.; Ozeki, T.; Nishimura, J.; Kayatani, H.; Minami, D.; Shinno, Y.; Sato, K.; et al. Rapidly progressive acute kidney injury associated with nivolumab treatment. Case Rep. Oncol. 2020, 13, 85-90. [CrossRef]

41. Tabei, A.; Watanabe, M.; Ikeuchi, H.; Nakasatomi, M.; Sakairi, T.; Kaneko, Y.; Maeshima, A.; Kaira, K.; Hirato, J.; Nojima, Y.; et al. The analysis of renal infiltrating cells in acute tubulointerstitial nephritis induced by anti-PD-1 antibodies: A case report and review of the literature. Intern. Med. 2018, 57, 3135-3139. [CrossRef]

42. Endo, N.; Tsuboi, N.; Furuhashi, K.; Shi, Y.; Du, Q.; Abe, T.; Hori, M.; Imaizumi, T.; Kim, H.; Katsuno, T.; et al. Urinary soluble CD163 level reflects glomerular inflammation in human lupus nephritis. Nephrol. Dial. Transplant. 2016, 31, 2023-2033. [CrossRef]

43. Villacorta, J.; Lucientes, L.; Goicoechea, E.; Acevedo, M.; Cavero, T.; Sanchez-Camara, L.; Díaz-Crespo, F.; Gimenez-Moyano, S.; García-Bermejo, L.; Fernandez-Juarez, G. Urinary soluble CD163 as a biomarker of disease activity and relapse in antineutrophil cytoplasm antibody-associated glomerulonephritis. Clin. Kidney J. 2021, 14, 212-219. [CrossRef]

44. Qualls, D.; Seethapathy, H.; Bates, H.; Tajmir, S.; Heidari, P.; Endres, P.; Reynolds, K.; Lawrence, D.; Sise, M. Positron emission tomography as an adjuvant diagnostic test in the evaluation of checkpoint inhibitor-associated acute interstitial nephritis. $J$. Immunother. Cancer 2019, 7, 356. [CrossRef]

45. Singh, P.; Brito, A.; Abdulnour, R.-E.E.; Grover, S.; Yenulevich, E.; Stuver, S.O.; Kehl, K.L.; LeBoeuf, N.R.; Jacobson, J.O.; Rahma, O.E. Defining real-world criteria for immune-related adverse events (irAEs). J. Clin. Oncol. 2019, 37, e14172. [CrossRef]

46. Gawai, P.P. Overview of important methods used for causality assessment of adverse drug events in pharmacovigilance. $J$. Pharmacovigil. Drug Res. 2020, 1, 6-11.

47. Shimamura, Y.; Watanabe, S.; Maeda, T.; Abe, K.; Ogawa, Y.; Takizawa, H. Incidence and risk factors of acute kidney injury, and its effect on mortality among Japanese patients receiving immune check point inhibitors: A single-center observational study. Clin. Exp. Nephrol. 2021, 25, 479-487. [CrossRef] [PubMed]

48. Scanvion, Q.; Béné, J.; Gautier, S.; Grandvuillemin, A.; Le Beller, C.; Chenaf, C.; Etienne, N.; Brousseau, S.; Cortot, A.B.; Mortier, L.; et al. Moderate-to-severe eosinophilia induced by treatment with immune checkpoint inhibitors: 37 Cases from a national reference center for hypereosinophilic syndromes and the French pharmacovigilance database. Oncoimmunology 2020, 9 , 1722022. [CrossRef]

49. Yılmaz, A.; Mirili, C.; Bilici, M.; Tekin, S.B. Possible atezolizumab-associated acute kidney injury and immune thrombocytopenia. J. Oncol. Pharm. Pract. 2020, 26, 1791-1794. [CrossRef] [PubMed]

50. Dumoulin, D.W.; Visser, S.; Cornelissen, R.; van Gelder, T.; Vansteenkiste, J.; von der Thusen, J. Aerts JGJV: Renal toxicity from pemetrexed and pembrolizumab in the era of combination therapy in patients with metastatic nonsquamous cell NSCLC. $J$. Thorac. Oncol. 2020, 15, 1472-1483. [CrossRef] [PubMed]

51. Hanna, R.M.; Selamet, U.; Bui, P.; Sun, S.F.; Shenouda, O.; Nobakht, N.; Barsoum, M.; Arman, F.; Rastogi, A. Acute Kidney Injury after Pembrolizumab-Induced Adrenalitis and Adrenal Insufficiency. Case Rep. Nephrol. Dial. 2018, 8, 171-177. [CrossRef] [PubMed]

52. Del Bello, A.; Zakaroff, A.G.; Meyer, N.; Delas, A.; Faguer, S.; Kamar, N.; Belliere, J. Cytokine storm induced by a PD1 inhibitor in a renal transplant patient. Am. J. Transplant. 2021. [CrossRef] [PubMed]

53. Manohar, S.; Ghamrawi, R.; Chengappa, M.; Goksu, B.N.B.; Kottschade, L.; Finnes, H.; Dronca, R.; Leventakos, K.; Herrmann, J.; Herrmann, S.M. Acute interstitial nephritis and checkpoint inhibitor therapy. Kidney360 2020, 1, 16-24. [CrossRef]

54. Fisher, J.; Zeitouni, N.; Fan, W.; Samie, F.H. Immune checkpoint inhibitor therapy in solid organ transplant recipients: A patient-centered systematic review. J. Am. Acad. Dermatol. 2020, 82, 1490-1500. [CrossRef]

55. Nguyen, L.S.; Ortuno, S.; Lebrun-Vignes, B.; Johnson, D.B.; Moslehi, J.J.; Hertig, A.; Salem, J.E. Transplant rejections associated with immune checkpoint inhibitors: A pharmacovigilance study and systematic literature review. Eur. J. Cancer 2021, 148, 36-47. [CrossRef]

56. Salem, J.E.; Allenbach, Y.; Kerneis, M. Abatacept for severe immune checkpoint inhibitor-associated myocarditis. N. Engl. J. Med. 2019, 380, 2377-2379. [CrossRef] [PubMed]

57. Herrmann, S.M.; Perazella, M.A. Immune checkpoint inhibitors and immune-related adverse renal events. Kidney Int. Rep. 2020, 5, 1139-1148. [CrossRef] [PubMed] 
58. Brahmer, J.R.; Drake, C.G.; Wollner, I.; Powderly, J.D.; Picus, J.; Sharfman, W.H.; Stankevich, E.; Pons, A.; Salay, T.M.; McMiller, T.L.; et al. Phase I study of single-agent anti-programmed death-1 (MDX-1106) in refractory solid tumors: Safety, clinical activity, pharmacodynamics, and immunologic correlates. J. Clin. Oncol. 2010, 28, 3167-3175. [CrossRef] [PubMed]

59. Martins, F.; Sykiotis, G.P.; Maillard, M.; Fraga, M.; Ribi, C.; Kuntzer, T.; Michielin, O.; Peters, S.; Coukos, G.; Spertini, F.; et al. New therapeutic perspectives to manage refractory immune checkpoint-related toxicities. Lancet Oncol. 2019, 20, e54-e64. [CrossRef]

60. Murakami, N.; Borges, T.J.; Yamashita, M.; Riella, L.V. Severe acute interstitial nephritis after combination immune-checkpoint inhibitor therapy for metastatic melanoma. Clin. Kidney J. 2016, 9, 411-417. [CrossRef] [PubMed]

61. Daanen, R.A.; Maas, R.J.H.; Koornstra, R.H.T.; Steenbergen, E.J.; Van Herpen, C.M.L.; Willemsen, A.E.C.A.B. Nivolumabassociated nephrotic syndrome in a patient with renal cell carcinoma: A case report. J. Immunother. 2017, 40, 345-348. [CrossRef]

62. Mamlouk, O.; Lin, J.S.; Abdelrahim, M.; Tchakarov, A.S.; Glass, W.F.; Selamet, U.; Buni, M.; Abdel-Wahab, N.; Abudayyeh, A. Checkpoint inhibitor-related renal vasculitis and use of rituximab. J. Immunother. Cancer 2020, 8, e000750. [CrossRef]

63. Bottlaender, L.; Breton, A.L.; Laforcade, L.; Dijoud, F.; Thomas, L.; Dalle, S. Acute interstitial nephritis after sequential ipilumumab-Nivolumab therapy of metastatic melanoma. J. Immunother. Cancer 2017, 5, 57. [CrossRef] [PubMed]

64. Tsung, I.; Worden, F.P.; Fontana, R.J. A pilot study of checkpoint inhibitors in solid organ transplant recipients with metastatic cutaneous squamous cell carcinoma. Oncologist 2021, 26, 133-138. [CrossRef] [PubMed]

65. Mejia, C.D.; Frank, A.M.; Singh, P.; Yadav, A. Immune checkpoint inhibitor therapy-associated graft intolerance syndrome in a failed kidney transplant recipient. Am. J. Transplant. 2021, 21, 1322-1325. [CrossRef] [PubMed] 